\title{
Trombose venosa profunda relacionada ao ciclo gravídico-puerperal e alterações fisiopatológicas com o advento do COVID-19
}

\author{
Deep venous thrombosis related to the pregnancy-puerperal cycle and physiopathological changes \\ with the advent of COVID-19 \\ Trombosis venosa profunda relacionada con el ciclo embarazo-puerperio y cambios fisiopatológicos \\ con el advenimiento del COVID-19
}

\author{
Mariana Tenório Costa \\ ORCID: https://orcid.org/0000-0001-8116-4864 \\ Centro Universitário Tiradentes, Brasil \\ E-mail: mtc0809@hotmail.com \\ Giovanna Mendonça Ferreira \\ ORCID: https://orcid.org/0000-0001-7634-4756 \\ Centro Universitário Tiradentes, Brasil \\ E-mail: giovanna-mendonca@hotmail.com \\ Lusitânia Maria de Barros \\ ORCID: https://orcid.org/0000-0001-6483-2832 \\ Centro Universitário Tiradentes, Brasil \\ E-mail: lusitaniabarros50@gmail.com
}

\begin{abstract}
Resumo
Objetivo: Avaliar os principais fatores de risco para Trombose Venosa Profunda (TVP) em pacientes gestantes e puérperas e se há relação do COVID-19 com elevação dos casos. Metodologia: Revisão sistemática de literatura, no qual foram realizadas buscas nas plataformas eletrônicas PubMed, LILACS e Scielo, utilizando os descritores: deep venous thrombosis, pregnancy e COVID-19. Resultados: A amostra resultou em 15 artigos. 93,3\% das pesquisas afirmam que mulheres no período gestacional ou puerperal apresentam maior probabilidade de desenvolver TVP devido a alterações fisiológicas do período, com $26,6 \%$ dos estudos totais demonstrando fatores de risco para piora do quadro, como cardiopatias, gestação em idade superior a 35 anos, parto cesárea, cirurgia recente entre outros. Não foi relatado em nenhum estudo o aumento de incidência dos casos de TVP em gestantes infectadas pelo vírus SARS-CoV-2, contudo $20 \%$ dos artigos demonstram o aumento do número de complicações associadas a infecção, como parto prematuro, aborto e óbito fetal. Conclusão: Há riscos elevados para eventos trombóticos na gestação, especialmente em gestantes com fatores de agrave, como os desencadeadores da tríade de Virchow (estase, hipercoagulação e dano vascular), com a necessidade de atenção especial a essas pacientes. Não há associação estabelecida entre aumento de casos de TVP com infecção por COVID-19, recomendado o uso profilático de Heparina de Baixo Peso Molecular (HBPM) nestas pacientes, embora sejam necessários mais estudos sobre o assunto em virtude do pouco tempo de aparecimento da doença.
\end{abstract}

Palavras-chave: Trombose Venosa Profunda; Gravidez; COVID-19.

\begin{abstract}
Objective: Evaluate the main risk factors for Deep Venous Thrombosis (DVT) in pregnant and postpartum patients and whether there is a relationship with COVID-19 with increased cases. Methodology: Systematic literature review, in which searches were performed on the electronic platforms PubMed, LILACS and Scielo, using the descriptors: deep venous thrombosis, pregnancy and COVID-19. Results: The sample resulted in 15 articles. $93.3 \%$ of the surveys state that women in the gestational or postpartum period are more likely to develop DVT due to physiological changes during the period, with $26.6 \%$ of the total studies showing risk factors for worsening the condition, such as heart disease, pregnancy and age older than 35 years, cesarean delivery, recent surgery, among others. No study reported an increased incidence of DVT cases in pregnant women infected with the SARS-CoV-2 virus, however $20 \%$ of the articles demonstrate an increase in the number of complications associated with infection, such as premature birth, abortion and fetal death. Conclusion: There are high risks for thrombotic events in pregnancy, especially in pregnant women with aggravating factors, such as the triggers of Virchow's triad (stasis, hypercoagulation and vascular damage), with the need for special attention to these patients. There is no established association between an increase in DVT cases with COVID-19 infection, and the prophylactic use of Low Molecular Weight Heparin (LMWH) is recommended in these patients, although more studies on the subject are needed due to the short time of disease onset.
\end{abstract}

Keywords: Deep Venous Thrombosis; Pregnancy; COVID-19. 


\begin{abstract}
Resumen
Objetivo: Evaluar los principales factores de riesgo de trombosis venosa profunda (TVP) en mujeres embarazadas, posparto y si hay relación entre COVID-19 y el aumento de casos. Metodología: Revisión sistemática de la literatura en la que se realizaron búsquedas en las plataformas electrónicas PubMed, LILACS y Scielo, utilizando los descriptores: deep venous thrombosis, pregnancy y COVID-19. Resultados: La muestra resultó en 15 artículos. El 93,3\% de las encuestas afirman que las mujeres en el período gestacional o posparto tienen más probabilidad de desarrollar TVP debido cambios fisiológicos durante el período, donde $26,6 \%$ de los estudios totales muestran factores de riesgo para empeorar el cuadro, como enfermedades cardíacas, embarazo en edad mayor de 35 años, parto por cesárea, cirugía reciente, además de otros. Ningún estudio informó una mayor incidencia de los casos de TVP en mujeres embarazadas infectadas con el virus SARS-CoV-2; sin embargo, el 20\% de los artículos demuestra un aumento en el número de complicaciones asociadas con la infección, como el parto prematuro, aborto y muerte fetal. Conclusión: Existen altos riesgos de eventos trombóticos del embarazo, especialmente en gestantes con factores agravantes, como los desencadenantes de la tríada de Virchow (estasis, hipercoagulación y daño vascular), con la necesidad de una atención especial a estas pacientes. No existe asociación establecida entre un aumento de los casos de TVP con infección por el COVID-19, y se recomienda el uso profiláctico de heparina con bajo peso molecular (HBPM) en estos pacientes, aunque, se necesitan más estudios sobre el tema debido al corto tiempo del inicio de la enfermedad.
\end{abstract}

Palabras clave: Trombosis de la Vena; Embarazo; COVID-19.

\title{
1. Introdução
}

Trombose venosa profunda (TVP), é uma das principais manifestações do tromboembolismo venoso (TEV), caracterizada pela formação de trombos, no compartimento profundo, provocando a obstrução total ou parcial do vaso. O desenvolvimento do trombo é dependente de alterações que foram descritas no século XIX por Virchow, como estase venosa, hipercoagulabilidade e lesão endotelial (Junior et al, 2013).

Como a TVP não desenvolve muitos sintomas específicos, a patologia pode rapidamente evoluir para embolia pulmonar (EP) e posteriormente para síndrome pós-trombótica, os quais são suas principais complicações, caso não seja diagnosticada e tratada precocemente (Barros et al, 2012).

Á visto disso, revela-se a importância de se atentar aos principais fatores de risco para poder ajudar no pensamento clínico para se diagnosticar uma TVP, como cirurgia prévia, imobilização recente, história prévia de tromboembolismo, gravidez, uso de hormônio terapia (estrogênio), a maioria sendo associado a dor e edema em membro inferior, alterações laboratoriais, principalmente com o D-dímero e imaginológicas como a ultrassonografia com doppler (USD) (Barros et al, 2012).

Em relação ao ciclo gravídico-puerperal, o risco de TEV chega a aumentar cerca de 5 a 10 vezes durante a gestação (sendo a TVP de membros inferiores equivalente a 75 a $80 \%$ destes casos), quando comparado a mulheres não gestantes, com casos em 1 a cada 1000 gestantes aproximadamente (Oliveira et al, 2016).

Na maioria dos casos, a TVP distribui-se de forma igualitária durante os 3 trimestres da gestação, com mais de $50 \%$ dos casos ocorrendo nos dois primeiros trimestres, e possui maior ocorrência no membro inferior esquerdo (90\%), quando comparado ao direito $(55 \%)$ e as mulheres não gestantes. No puerpério a frequência eleva, com chances cerca de 20 vezes maiores de eventos trombóticos, quando em comparação a mulheres pré-gestação, entretanto, após esse período, o risco de um possível TEV diminui (Oliveira et al, 2016).

O diagnóstico no ciclo gravídico-puerperal costuma ser ainda mais difícil, devido as modificações que ocorrem no organismo materno. Mulheres costumam queixar-se de condições clínicas que ocorrem durante esse período, entretanto, na maioria dos casos, as alterações são fisiológicas deste período, o quais são causadas por fatores hormonais e mecânicos, não ocasionando preocupações acerca da saúde da paciente. Todavia, a minoria, como alterações sanguíneas, deve ser observada de perto pelo médico para evitar uma complicação obstétrica direta. (Montenegro \& Rezende, 2018).

Em Março de 2020, com o advento da pandemia causada pelo vírus SARS-CoV-2, observou-se alto potencial trombogênico. A fisiopatologia do vírus envolve uma infecção, relacionada aos receptores ECA II presente em muitas superfícies celulares. Com uma resposta inflamatória importante, há ativação macrofágica e de citocinas pró inflamatórias, que 
posteriormente levam a dano endotelial e ativação plaquetária. A maioria dos pacientes são assintomáticos ou com sintomas leves, apenas 20\% evoluindo para casos graves. Quando há TEV associado ao vírus, (Coagulopatia Associada a COVID-19, ou $\mathrm{CAC}$ ) ocorre um aumento da mortalidade em cerca de 2x mais comparado às pessoas que não adquiriram a doença (Freire $e t$ al, 2020).

Os efeitos da infecção pelo vírus SARS-CoV-2 em gestantes podem ser decorrentes do efeito viral ou das respostas hiperinflamatórias. Infecções severas induzem mecanismos imunes, além de hipercoagulação, devido a ativação da cascata de coagulação, que pode causar trombo ou consumo de fatores, com manifestações que podem ser hemorrágicas ou trombóticas. (Ferrer-Oliveras et al, 2021).

A TVP representa uma importante causa de morbimortalidade em pacientes gestantes e o advento da infecção por SARS-CoV-2 tem fisiopatologia associada a alterações de coagulação. Dessa forma, o presente estudo tem como objetivo avaliar, através de uma revisão sistemática da literatura, os principais fatores de risco para Trombose Venosa Profunda em pacientes gestantes e puérperas e se há relação de COVID-19 com elevação de casos.

\section{Metodologia}

Trata-se de um estudo baseado no método de revisão sistemática de literatura, no qual consiste em uma produção científica a partir de estudos já publicados. De acordo com Roever (2017), é frequentemente utilizado para análise de conceitos, teorias e evidências sobre determinado tema, além de identificar ausência de informações que necessitam ser complementadas em novos estudos, com finalidade de maior compreensão do assunto.

A produção da pesquisa, realizada durante o período de Janeiro a Outubro de 2021, iniciou-se com a delimitação do tema e a formulação da pergunta norteadora, seguido da coleta de pesquisas de fontes secundárias, através de bases de dados bibliográficos eletrônicos como LILACS (Literatura Latino-Americana e do Caribe em Ciências da saúde), PubMed, e Scielo (Scientific Eletronic Library Online). Após a pesquisa, os estudos foram avaliados, com resultados interpretados e sintetizados.

A pergunta norteadora do estudo foi "Quais as implicações da trombose venosa profunda em mulheres grávidas e puérperas e possíveis interferências da infecção por COVID-19 a esse grupo de risco?”. Com o objetivo de responder o questionamento, foi utilizado um planejamento de pesquisa nas plataformas citadas anteriormente.

Em cada plataforma de pesquisa, foi utilizado o termo bolleano AND para cruzamento de achados e como descritores: "deep venous thrombosis", "pregnancy" e "covid-19" nas línguas portuguesa e inglesa, com diferentes combinações.

Como critérios de inclusão, foram utilizados artigos que respondessem à pergunta norteadora, disponíveis na íntegra, publicados nos últimos 10 anos, entre os anos de 2011 e 2021, em todas as categorias de artigo, nos idiomas português, inglês e espanhol, prévios e após a pandemia pelo COVID-19. Já como critérios de exclusão, foram descartados artigos duplicados, como também os que não tiveram relação direta com o objetivo da pesquisa.

Após os critérios pré-definidos, foi realizado uma leitura criteriosa para analisar quais artigos iriam compor a pesquisa (Figura 1). 
Figura 1. Identificação e seleção dos artigos.

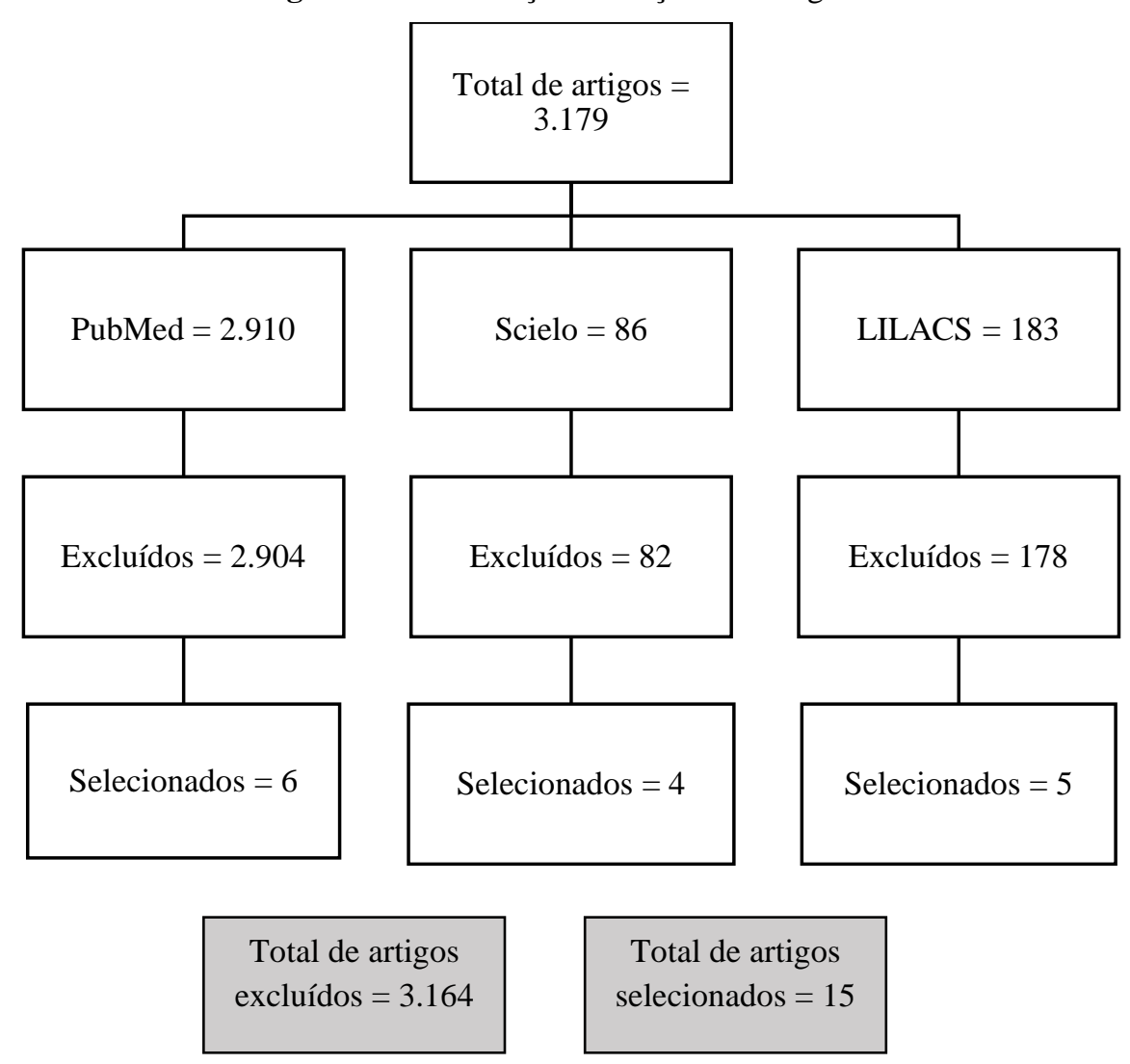

Fonte: Dados da pesquisa no PubMed, Scielo e LILACS até outubro de 2021.

Em um universo de 3.179 artigos, 3.164 foram excluídos e 15 foram selecionados como amostra para compor a pesquisa (Figura 1).

\section{Resultados}

Os artigos encontrados foram analisados de acordo com o título, procedência, tipo de estudo e principais achados (Tabela 1). Levando em consideração os critérios de seleção, foram observados os seguintes resultados:

Tabela 1. Resultados da pesquisa.

\begin{tabular}{|l|c|l|l|l|}
\hline Título do artigo & Autores & Procedência & $\begin{array}{c}\text { Tipo de } \\
\text { Estudo }\end{array}$ & Principais achados \\
\hline $\begin{array}{l}\text { Eventos tromboembólicos na } \\
\text { gestação e puerpério: revisão } \\
\text { sistemática e recomendação atual }\end{array}$ & $\begin{array}{c}\text { Pontes } \text { et } \\
\text { al. }\end{array}$ & LILACS & $\begin{array}{c}\text { Revisão } \\
\text { sistemática }\end{array}$ & $\begin{array}{l}\text { TVP possui uma morbidade significativa na gravidez, } \\
\text { devendo, dessa forma, estar atento aos fatores de risco } \\
\text { para o desenvolvimento de TEV }\end{array}$ \\
\hline $\begin{array}{l}\text { Enfermedad tromboembólica venosa } \\
\text { en el embarazo y puerperio. Enfoque } \\
\text { de riesgo y diagnóstico }\end{array}$ & $\begin{array}{c}\text { Mendez \& } \\
\text { Nunez }\end{array}$ & LILACS & $\begin{array}{c}\text { Revisão } \\
\text { narrativa }\end{array}$ & $\begin{array}{l}\text { O TEV requer a identificação precoce para realizar o } \\
\text { tratamento e ter uma rápida estabilização dos sintomas } \\
\text { da gestante }\end{array}$ \\
\hline $\begin{array}{l}\text { Thrombosis in Coronavirus disease } \\
\text { 2019 (COVID-19) through the prism } \\
\text { of Virchow's triad }\end{array}$ & $\begin{array}{c}\text { Ahmed } \text { et } \\
\text { al. }\end{array}$ & PubMed & $\begin{array}{l}\text { Revisão } \\
\text { narrativa }\end{array}$ & $\begin{array}{l}\text { A fisiopatologia do Covid-19 explica a sua alta } \\
\text { propensão para o desenvolvimento de trombose }\end{array}$ \\
\hline Novel coronavirus infection and & Yang et al. & PubMed & Revisão & Não há evidências que grávidas sejam mais suscetíveis \\
\hline
\end{tabular}




\begin{tabular}{|c|c|c|c|c|}
\hline pregnancy & & & narrativa & à infecção por covid-19 \\
\hline $\begin{array}{l}\text { COVID-19 y embarazo: Una } \\
\begin{array}{l}\text { aproximación en tiempos } \\
\text { pandemia }\end{array}\end{array}$ & $\begin{array}{l}\text { Cardonne } \\
\quad \text { et al. }\end{array}$ & Scielo & $\begin{array}{l}\text { Revisão } \\
\text { narrativa }\end{array}$ & $\begin{array}{l}\text { A clínica e a suscetibilidade de desenvolver covid-19 } \\
\text { em gestantes não é diferente das mulheres não grávidas }\end{array}$ \\
\hline $\begin{array}{l}\text { SEDAR-SEMICYUC consensus } \\
\text { recommendations on the } \\
\text { management of haemostasis } \\
\text { disorders in severely ill patients with } \\
\text { COVID-19 infection }\end{array}$ & Llau et al. & Pubmed & $\begin{array}{l}\text { Revisão } \\
\text { narrativa }\end{array}$ & $\begin{array}{l}\text { A infecção por COVID eleva fatores pró coagulantes, } \\
\text { com alteração frequente de fibrinogênio plasmático, } \\
\text { que pode evoluir para Coagulação Intravascular } \\
\text { Disseminada (CIVD). Foram calculados } 8,7 \% \text { dos } \\
\text { casos com CIVD associada a COVID. }\end{array}$ \\
\hline $\begin{array}{l}\text { Physician experiences in } \\
\text { management of COVID-19- } \\
\text { associated coagulopathy in } \\
\text { pregnancy: Communication from the } \\
\text { ISTH SSC Subcommittee on } \\
\text { Women's Health Issues in } \\
\text { Thrombosis and Haemostasis }\end{array}$ & $\begin{array}{l}\text { Jevtic, et } \\
\quad \text { al. }\end{array}$ & Pubmed & $\begin{array}{l}\text { Estudo } \\
\text { descritivo }\end{array}$ & $\begin{array}{l}1 \% \text { dos casos de COVID desenvolvem Coagulopatia } \\
\text { Associada a COVID-19 (CAC). As anormalidades } \\
\text { incluem principalmente PCR elevado, D dímero } \\
\text { elevado e linfopenia. Cerca de } 7,35 \% \text { de casos de CAC } \\
\text { são trombóticos }\end{array}$ \\
\hline $\begin{array}{l}\text { Venous sinus thrombosis during } \\
\text { COVID-19 infection in pregnancy: a } \\
\text { case report }\end{array}$ & Gunduz. & Pubmed & $\begin{array}{l}\text { Relato de } \\
\text { Caso }\end{array}$ & $\begin{array}{l}\text { Além da fisiopatologia relacionada a CIVD induzida } \\
\text { por SEPSE, o COVID também possui relação com a } \\
\text { microangiopatia trombótica mediada por } \\
\text { complemento, que aumenta a chance de eventos } \\
\text { trombóticos }\end{array}$ \\
\hline $\begin{array}{l}\text { Haemostatic and thrombo-embolic } \\
\text { complications in pregnant women } \\
\text { with COVID-19: a systematic } \\
\text { review and critical analysis }\end{array}$ & $\begin{array}{l}\text { Servante, } \\
\quad \text { et al. }\end{array}$ & Pubmed & $\begin{array}{l}\text { Revisão } \\
\text { sistemática }\end{array}$ & $\begin{array}{l}\text { De } 1263 \text { pacientes, } 3 \text { tiveram TVP ou Trombose } \\
\text { Arterial, } 7 \text { tiveram CIVD. Pela elevação do risco de } \\
\text { trombose, é recomendado o uso profilático de Heparina } \\
\text { de Baixo Peso Molecular (HBPM), a menos que o parto } \\
\text { esteja previsto em } 12 \mathrm{~h} \text {. }\end{array}$ \\
\hline $\begin{array}{l}\text { Trombosis venosa profunda de } \\
\text { miembro inferior en gestante del } \\
\text { primer trimestre. Reporte de caso }\end{array}$ & $\begin{array}{l}\text { Ramirez- } \\
\text { Calderon } \\
\quad \text { et al. }\end{array}$ & Scielo & $\begin{array}{l}\text { Relato de } \\
\text { Caso }\end{array}$ & $\begin{array}{l}\text { TVP é uma importante causa de morbimortalidade em } \\
\text { gestantes, com incidência relacionada a gestação de } 1 \\
\text { caso a cada } 1.000 \text { gestações. Cerca de } 20 \% \text { dos casos } \\
\text { de TVP sem TEP são relacionados a Síndrome Anti- } \\
\text { fosfolípide }\end{array}$ \\
\hline $\begin{array}{l}\text { Tromboembolia pulmonar en el } \\
\text { embarazo y puerpério }\end{array}$ & $\begin{array}{l}\text { Roja- } \\
\text { Sanchez, } \\
\text { et al. }\end{array}$ & Scielo & $\begin{array}{l}\text { Revisão } \\
\text { narrativa }\end{array}$ & $\begin{array}{l}\text { Durante o parto existe lesão vascular e adjacências na } \\
\text { interfase útero-plaquetária, que exacerba durante o } \\
\text { parto cesárea. A cascata de coagulação é ativada e, } \\
\text { depois do nascimento se iniciam reações de fase aguda, } \\
\text { com antitrombina III elevada até } 1^{\text {a }} \text { semana do } \\
\text { puerpério }\end{array}$ \\
\hline $\begin{array}{l}\text { Experiencia de } 11 \text { años en la } \\
\text { enfermedad tromboembólica venosa } \\
\text { en el período grávido puerperal }\end{array}$ & $\begin{array}{l}\text { Mendez \& } \\
\text { Nunes }\end{array}$ & Scielo & $\begin{array}{l}\text { Estudo } \\
\text { descritivo }\end{array}$ & $\begin{array}{l}84,1 \% \text { dos casos de TVP na gestação foram no período } \\
\text { anteparto e } 15,9 \% \text { no puerpério, sendo a manifestação } \\
\text { clínica mais comum a dor }(98,6 \%) \text { e fator de risco mais } \\
\text { comum a presença de varizes }(36,5 \%)\end{array}$ \\
\hline $\begin{array}{l}\text { Período pós-parto e infecção pelo } \\
\text { novo coronavírus: revisão de escopo }\end{array}$ & $\begin{array}{l}\text { Oliveira, } \\
\text { et al. }\end{array}$ & LILACS & $\begin{array}{l}\text { Revisão de } \\
\text { escopo }\end{array}$ & $\begin{array}{l}\text { Mulheres no pós parto estão mais suscetíveis a } \\
\text { infecções, devido a hormônios que tentam compensar } \\
\text { o estresse do parto. Sugere-se que o risco de formação } \\
\text { de trombos, visto o efeito do COVID na coagulação e } \\
\text { o estado de hipercoagulabilidade na gestação, } \\
\text { especialmente em partos cesárea e gestantes com } \\
\text { comorbidades. }\end{array}$ \\
\hline $\begin{array}{l}\text { Influencia de la COVID-19 en el } \\
\text { embarazo desde la perspectiva de los } \\
\text { cuidado }\end{array}$ & $\begin{array}{l}\text { Gámez- } \\
\text { Gámez, et } \\
\quad \text { al. }\end{array}$ & LILACS & $\begin{array}{l}\text { Revisão } \\
\text { narrativa }\end{array}$ & $\begin{array}{l}\text { Mulheres gestantes são afetadas de forma mais severa } \\
\text { em infecções respiratórias. Na infecção pelo SARS- } \\
\text { CoV02 ocorreria uma tempestade de citocinas e } \\
\text { substâncias pró-inflamatórias que podem induzir } \\
\text { resposta exagerada, principalmente no primeiro e } \\
\text { terceiro trimestres. As gestantes possuem mais riscos } \\
\text { de CIVD. }\end{array}$ \\
\hline
\end{tabular}




\begin{tabular}{|c|c|c|c|c|}
\hline $\begin{array}{l}\text { Posicionamento sobre COVID-19 e } \\
\text { Gravidez em Mulheres Cardiopatas - } \\
\text { Departamento de Cardiologia da } \\
\text { Mulher da Sociedade Brasileira de } \\
\text { Cardiologia - } 2020\end{array}$ & $\begin{array}{c}\text { Marques- } \\
\text { Santos, et } \\
\text { al. }\end{array}$ & LILACS & $\begin{array}{c}\text { Revisão } \\
\text { narrativa }\end{array}$ & $\begin{array}{l}\text { Distúrbios de coagulação são bastante importantes na } \\
\text { morbimortalidade de COVID-19, pois a infecção tem } \\
\text { característica pró-trombótica com aumento de fibrina, } \\
\text { de seus produtos, do fibrinogênio e D-dímero. } \\
\text { Combinada com a gestação e cardiopatias aumenta o } \\
\text { risco da formação de trombos, especialmente arteriais, } \\
\text { requerendo esquema de anticoagulação. }\end{array}$ \\
\hline
\end{tabular}

Fonte: Dados da pesquisa no PubMed, Scielo e LILACS até outubro de 2021.

Dentre os estudos selecionados, disponíveis na Tabela 1, a maioria, 53,3\% $(\mathrm{n}=8)$, foi caracterizado como revisão narrativa, $13,3 \%(\mathrm{n}=2)$ foram identificados como relatos de caso, 13,3\% $(\mathrm{n}=2)$ como revisão sistemática, 13,3\% $(\mathrm{n}=2)$ como estudo descritivo e por fim, apenas 6,6\% $(\mathrm{n}=1)$ como estudo de escopo. Quanto à procedência dos artigos, 40\% (n = 6) são originários do PubMed, 33,3\% ( $\mathrm{n}=5)$ são do LILACS e 26,6\% ( $\mathrm{n}=4)$ do Scielo.

Foi observado que em $93,3 \%$ das pesquisas $(n=14)$, as mulheres quando estão grávidas ou no puerpério, possuem maior probabilidade de desenvolver TVP devido às alterações de coagulação que ocorrem no organismo materno, com a ativação da tríade de Virchow.

Já em 26,6\% dos estudos $(n=4)$, é citado os principais fatores de risco para eventos trombóticos nesse período, com destaque para Mendez \& Nunes (2011) que citam varizes em membros inferiores em cerca de 36,5\% dos casos, repouso prolongado em 35,8\% e uso prévio de anticonceptivos orais em 29,6\% do grupo. Marques-Santos, et al (2020) apresentam que a combinação entre gestação, infecção por COVID-19 e cardiopatias pressupõe-se que eleva as chances de eventos trombóticos, principalmente o tromboembolismo arterial.

Todavia, nessa mesma análise de indivíduos, dentre os estudos da amostra, não foi relatado discrepância quanto ao aumento de número de casos de TVP em mulheres que estão com Covid-19 no ciclo gravídico-puerperal, comparado a grávidas sem a infecção.

Na relação entre Covid-19 e gestantes e/ou puérperas, Marques-Santos et al (2020) e Oliveira et al (2021), sugerem que a combinação covid-19 e gravidez aumentam o risco e a probabilidade do desenvolvimento do tromboembolismo venoso devido ao estado de hipercoagulabilidade, principalmente no puerpério, entretanto, dentre as patologias que envolvem TEV, não há citações sobre a TVP.

Em coadunação com o estudo publicado por Servante et al (2021), de 1.063 mulheres com SARS-CoV-2, 3 foram diagnosticadas com tromboembolismo, 7 com CIVD e outras 3 com coagulopatia, indicando que as complicações hematológicas são mais comumente observadas em mulheres grávidas por Covid-19 (1,26\%) do que em mulheres grávidas sem a infecção $(0,45 \%)$.

Já os estudos de Cardonne et al (2020), Gamez-Gamez et al (2021), Marques-Santos et al (2020) e Yang et al (2020), os quais correspondem a $26,6 \%$ da amostra, apontam que há maior probabilidade destas desenvolverem infecções respiratórias devido às alterações fisiológicas que ocorrem no organismo materno.

Além disso, $20 \%$ dos estudos $(n=3)$, ainda relatam aumento do número de complicações obstétricas quando também associado ao Covid-19, como pré-eclâmpsia, restrição do crescimento fetal, rotura prematura das membranas, parto prematuro, aborto e óbito fetal.

Por fim, 53,3\% dos estudos $(\mathrm{n}=8)$ indicam que deve ser realizado o tratamento em pacientes gestantes e puérperas que possuem risco para eventos trombóticos, sendo a heparina de baixo peso molecular a escolha. Destes, 4 pesquisas (26,6\%), indicaram que nessas mulheres, mesmo infectadas com Covid-19, deve ser realizada a profilaxia com o mesmo anticoagulante. 


\section{Discussão}

Como foi observado nos estudos, a TVP, mesmo não sendo um evento comum, é uma importante causa de morbimortalidade e possui maior incidência quando associada ao ciclo gravídico-puerperal, com incidência de aproximadamente 1 caso a cada 1.000 - 2.000 gestações. Ramirez-Calderon et al (2018) explana que o diagnóstico é ainda mais difícil de ocorrer nesse período devido às adaptações maternas que se desenvolvem durante toda a gravidez, simulando sinais clínicos como edema e desconforto em membros inferiores, todavia, existem alterações fisiológicas os quais favorecem o acometimento dessa patologia na mulher.

Ramirez-Calderon et al (2018) esclarece que durante a gravidez e o puerpério, tais alterações são decorrentes da ativação da tríade de Virchow, no qual fundamenta-se em hipercoagulabilidade, estase venosa e lesão endotelial.

A hipercoagubilidade consiste no aumento dos fatores de coagulação II, VII, VIII, X e o de von Willebrand, associado a diminuição de atividade fibrinolítica e aumento de agregação plaquetária, dessa forma, contribui para o estado pró-trombótico, principalmente no $3^{\circ}$ trimestre da gestação (Pontes et al, 2013).

Já a estase venosa, é caracterizada como uma redução de até 50\% do fluxo venoso dos membros inferiores, no qual ocorre a partir da $25^{\mathrm{a}}$ semana, alcançado um pico na $36^{\mathrm{a}}$ semana e somente voltando a ter um fluxo sanguíneo normal em 6 a 8 semanas pós-parto (após puerpério), devido a compressão do útero gravídico sobre a veia ilíaca esquerda, consequentemente, há uma maior predisposição (cerca de 70 a 90\%) pela trombose em membro inferior esquerdo (Pontes et al, 2013).

Por fim, a disfunção endotelial costuma ocorrer durante o parto (sendo ele vaginal ou cesáreo) e ocasiona a diminuição da atividade fibrinolítica, o que predispõem a formação de trombos (Ahmed et al, 2020). Nesse processo, Roja-Sanchez et al (2014) afirma que a cascata de coagulação é ativada e se iniciam as reações de fase aguda, com antitrombina III elevada até a $1^{\mathrm{a}}$ semana do puerpério.

Além disso, Pontes et al (2013) e Ramirez-Calderon et al (2018) citam fatores de risco que quando associados ao ciclo gravídico-puerperal, aumentam a probabilidade do desenvolvimento da patologia, como idade avançada (maior que 35 anos), obesidade, trombofilias (ex.: síndrome do anticorpo antifosfolipídeo), imobilidade, cirurgia recente, e história prévia de TVE. Bem como, existem os fatores de risco obstétricos, os quais são parto cesárea, parto prematuro ( 36 semanas), morte fetal, tabagismo, hipertensão sistêmica crônica e pré-eclâmpsia ou eclampsia.

Dessa forma, é de tamanha importância do médico obstetra, quando suspeitar da patologia, atentar-se a tais fatores, além de associá-los com a clínica da paciente, exame imaginológico e o escore de Wells. Mendez e Nunez (2011) reiteram que o diagnóstico não é realizado de forma isolada, sendo necessário correlacionar essas condições citadas anteriormente.

Nos estudos estabelecidos como amostra para Mendez e Nunez (2011), foi observado que o quadro clínico é caracterizado pelo aparecimento de edema, geralmente unilateral, na perna afetada, acompanhada de dor local e sinais flogísticos, como sensação de calor e eritema. Ademais, podem ser relatadas dores abdominais baixas, devido a extensão proximal com a veia pélvica.

Mendez e Nunes (2011) também relatam sobre a importância do exame físico, através de manobras, que auxiliam na avaliação do sistema venoso nos membros inferiores, como o sinal de Homans, identificado como dor na panturrilha a dorsiflexão do pé (ocorre a compressão das veias trombosadas pelos músculos da panturrilha), sinal da Bandeira, caracterizado pela menor mobilidade, durante a palpação, da panturrilha acometida, (indicando o empastamento) e por fim, o sinal de Olow, definido como dor a compressão da panturrilha sobre um plano ósseo.

Dentre os exames imaginológicos disponíveis, como venografia e pletismografia, a ultrassonografia doppler (USD), dos sistemas venosos superficial e profundo, é o método padrão-ouro para auxiliar no diagnóstico, por ser um exame não invasivo, não utilizar contraste e ter boa relação custo-benefício. Costuma ter mais de $95 \%$ de sensibilidade para detectar trombos poplíteos, femorais e ilíacos, com possibilidade de identificação de trombos distais em até 50\% dos casos, podendo ser realizado 
conjuntamente com a manobra de Valsalva. Assim, os estudos de Mendez e Nunez (2011) e Ramirez-Calderon et al (2018), estabelecem o USD como sendo o exame de escolha para a avaliação.

Todavia, é um exame operador-dependente, ou seja, para se ter um resultado preciso e confiável, é recomendado que o examinador tenha um treinamento específico e adequado para um bom manejo do aparelho, além do conhecimento anatômico da região, pois, no segundo trimestre da gravidez esses procedimentos devem ser realizados com o útero deslocado lateralmente (devido a compressão das veias ilíaca e cava, o que pode gerar resultados falso-positivos), caso contrário, o exame pode não ser 100\% fidedigno a condição do paciente (Méndez \& Núnez, 2011).

Já o critério de Wells, citado por Méndez e Núnez (2011) e Rojas-Sanchez et al (2014), é um escore que avalia a probabilidade de um paciente evoluir para TEP (principal complicação da TVP). Utiliza os critérios e pontos demonstrados na Tabela 2.

Tabela 2. Critérios de Wells.

\begin{tabular}{|l|c|}
\hline \multicolumn{1}{|c|}{ Critérios } & Pontos \\
\hline Suspeita de TEV & 3 pontos \\
\hline $\begin{array}{l}\text { Alternativa menos provável que } \\
\text { embolia pulmonar }\end{array}$ & 3 pontos \\
\hline Frequência cardíaca > 100 bpm & 1,5 pontos \\
\hline $\begin{array}{l}\text { Imobilização ou cirurgia nas } \mathbf{4} \\
\text { semanas anteriores }\end{array}$ & 1,5 pontos \\
\hline TEV prévio & 1,5 pontos \\
\hline Hemoptise & 1 ponto \\
\hline Carcinoma & 1 ponto \\
\hline
\end{tabular}

Fonte: Adaptação de Méndez e Núnez (2011).

A interpretação da Tabela 2 é feita analisando e somando a pontuação do paciente. É caracterizada como baixa probabilidade de desenvolver TEP quando tem de 0 a 2 pontos, moderada probabilidade com 3 a 6 pontos e alta probabilidade com mais de 6 pontos.

Além disso, dentre os exames laboratoriais, existe um que é muito utilizado na prática clínica quando se suspeita de trombos, porém Mendez e Nunez (2011) afirmam ser controverso quando se tem uma paciente grávida. Tal exame é o D-dímero, um produto da degradação da fibrina e possui níveis elevados em pessoas com trombose. Em coadunação com Marques-Santos et al (2020), seu uso é questionável na gravidez, visto que o valor é maior para o ponto de corte convencional (500 $\mu \mathrm{g} / \mathrm{L}) \mathrm{em} 99 \%$ das gestantes saudáveis. Assim, quando é utilizado, é mais devido ao seu alto valor preditivo negativo, que compreende cerca de $90 \%$, quando a patologia não está presente.

A respeito sobre a atual pandemia, considera-se que o SARS-CoV-2 pertence à família Coronaviridae e subgrupo de beta coronavírus, sendo transmitido de pessoa-pessoa através de secreções respiratórias por aerossóis. A infecção por COVID19 leva a uma reação inflamatória generalizada, com tempestade de citocinas e substâncias pró-inflamatórias, essas com possibilidade de induzir reação mais exagerada que pode desencadear Coagulação Intravascular Disseminada (CIVD), principalmente no primeiro e terceiro trimestres da gestação, devido a alterações imunológicas da gravidez e em gestantes com riscos prévios. (Gámez-Gámez et al, 2021).

Imunologicamente, Cardonne et al (2020) e Gámez-Gámez et al (2021), identificaram, de forma similar, as adaptações que ocorrem no organismo materno. $\mathrm{O} 1^{\circ}$ trimestre é caracterizado por um estado pró-inflamatório em vista de favorecer a invasão trofoblástica, já no $2^{\circ}$ trimestre, é desenvolvido uma resposta anti-inflamatória para beneficiar o crescimento fetal e prevenir o início espontâneo do trabalho de parto, por último, no $3^{\circ}$ trimestre, o organismo retorna ao estado pró-inflamatório, 
para ocorrer a preparação do parto. Dessa forma, em teoria, as pacientes grávidas, nos estágios pró-inflamatórios, teriam uma maior probabilidade de desenvolver a tempestade de citocinas, que é um indicador de gravidade na infecção pelo Covid-19.

Distúrbios de coagulação são um ponto crucial da morbimortalidade do COVID-19, pois está associada a inflamação e estado pró-trombótico, com elevação de fibrina, seus produtos de degradação, fibrinogênio e D-dímero, provavelmente em decorrência a uma resposta exagerada do organismo a infecção (sepse), contudo, Gunduz (2021) afirma que o quadro também pode possuir relação com a microangiopatia trombótica mediada por complemento, que aumentaria as chances de eventos trombóticos, Segundo Jevtic et al (2021), cerca de 1\% dos casos evoluem com Coagulopatia Associada a COVID (CAC).

Segundo Yang et al (2020), as gestantes são mais suscetíveis a infecções, especialmente respiratórias, pois ocorre alteração da imunidade dos linfócitos T, aumento do consumo de oxigênio, diminuição da capacidade residual funcional e diminuição da complacência torácica.

No estudo de Gámez-Gámez et al (2021), que ocorreu em Cuba, foi observado que cerca de $86 \%$ das gestantes apresentaram quadros leves de COVID-19, enquanto $14 \%$ apresenta quadros graves, destes $75 \%$ com sintomas similares a préeclâmpsia, hemólise e alterações hepáticas, além de síndrome de HELLP, com resolução espontânea após a infecção, bem como maiores chances de parto cesárea. As maiores complicações forem maiores riscos de aborto, rotura prévia de placenta e óbito fetal.

Dessa forma, de acordo com Llau et al (2020), com a fisiopatologia da infecção envolvendo a hipercoagulabilidade, acreditava-se que mulheres grávidas infectadas pelo novo coronavírus seriam ainda mais propensas a desenvolver manifestações tromboembólicas venosas, principalmente no puerpério, momento em que estão mais suscetíveis a infecção. Todavia, em estudos realizados nos Estados Unidos e no Reino Unido, não foi apontado um aumento de casos de TEV em mulheres grávidas com Covid-19, em relação a pacientes não grávidas infectadas (D’Souza et al, 2020).

Quanto ao tratamento da TVP, a heparina de baixo peso molecular (HBPM) é o anticoagulante de primeira linha, inclusive em mulheres que estão no ciclo gravídico-puerperal, pois o mesmo não atravessa a placenta, e dessa forma, não causa efeito teratogênico e hemorrágico ao feto (Pontes et al, 2013). Segundo Llau et al (2020) e Servante et al (2021), em pacientes infectadas com COVID-19, é recomendado o uso profilático da HBPM, a fim de diminuir os riscos de eventos adversos trombóticos.

\section{Conclusão}

No tocante aos resultados avaliados pelas pesquisas produzidas entre 2011 a 2021, há uma concordância entre os artigos analisados em relação à condição clínica de uma mulher gestante ou no puerpério, obter maiores riscos para desenvolver uma TVP. Já em relação a essa mesma amostra, quando associada a infecção pelo Covid-19, ainda não há uma associação estabelecida e estudos com evidência o suficiente nessas pacientes que indique o aumento do número de casos com trombose venosa profunda, sendo necessários mais pesquisas sobre o assunto em virtude do pouco tempo de existência da patologia. Ainda assim, é recomendado profilaxia com uso de heparina de baixo peso molecular para gestantes infectadas.

\section{Referências}

Ahmed, S., Zimba, O., \& Gasparyan, A. Y. (2020). Thrombosis in Coronavirus disease 2019 (COVID-19) through the prism of Virchow's triad. Clinical Rheumatology, 39(9), 2529-2543.

Barros, M. V. L., Pereira, V. S. R., \& Pinto, D. M. (2012). Controvérsias no diagnóstico e tratamento da trombose venosa profunda pela ecografia vascular. Jornal Vascular Brasileiro, 11(2), 137-143.

Cardonne, C. T. M., Cantillo, K. M., Durades, T. M. P., \& Lora, L. D. V. (2020). COVID-19 y embarazo: Una aproximación en tiempos de pandemia. MEDISAN, 24(4), 707-727. 
D'Souza, R., Malhamé, I., Teshler, L., Acharya, G., Hunt, B. J., \& McLintock, C. (2020). A critical review of the pathophysiology of thrombotic complications and clinical practice recommendations for thromboprophylaxis in pregnant patients with COVID-19. Acta obstetricia et gynecologica Scandinavica, 99(9), $1110-1120$.

Ferrer-Oliveras, R., Mendoza, M., Capote, S., Pratcorona, L., Esteve-Valverde, E., Cabero-Roura, L., \& Alijotas-Reig, J. (2021). Immunological and physiopathological approach of COVID-19 in pregnancy. Archives of gynecology and obstetrics, 304(1), 39-57.

Freire, J. F. B., Flores, S. S. T., \& Guerrero, M. A. B. (2021). SARS-CoV-2 y enfermedad tromboembólica venosa. No todo es nuevo. Angiología, 72(6), 286297.

Gámez-Gámez, L. N., Gámez-Borges, L. N., José-Sorrillo, L., \& Quiala, H. A. M. (2021). Influencia de la COVID-19 en el embarazo desde la perspectiva de los cuidados intensivos. Revista Informacion Cientifica, 100(4), e3351.

Gunduz Z. B. (2021). Venous sinus thrombosis during COVID-19 infection in pregnancy: a case report. Sao Paulo Medical Journal, 139 (2), 190-195.

Jevtic, S. D., Malinowski, A. K., Othman, M., \& Kadir, R. A. A. (2021). Physician experiences in management of COVID- 19- associated coagulopathy in pregnancy: Communication from the ISTH SSC Subcommittee on Women's Health Issues in Thrombosis and Haemostasis. J. Thromb. Haemost. 19, $2539-2545$.

Junior, N. M., Kingerski, F. N. M., Marioto, G. L., Viegas, F. A. F., Mesquita, S. F. S., \& Perreto, S. (2013). Prevalência de trombose venosa profunda em paraplégicos de causa traumática. Jornal Vascular Brasileiro, 12(4), 271-277.

Llau, J. V., Ferrandis, R., Sierra, P., Hidalgo, F., Cassinello, C., Gómez-Luque, A., Quintana, M., Amezaga, R., Gero, M., Serrano, A., \& Marcos, P. (2020). SEDAR-SEMICYUC consensus recommendations on the management of haemostasis disorders in severely ill patients with COVID-19 infection. Recomendaciones de consenso SEDAR-SEMICYUC sobre el manejo de las alteraciones de la hemostasia en los pacientes graves con infección por COVID19. Revista espanola de anestesiologia y reanimacion, 67(7), 391-399.

Marques-Santos, C., Avila, W. S., Carvalho, R. C. M., Lucena, A. J. G., Freire, C. M. V., Campanharo, F. F., Rivera, M. A. M. R., Costa, M. E. N. C., \& Castro, M. L. (2020). Posicionamento sobre COVID-19 e Gravidez em Mulheres Cardiopatas - Departamento de Cardiologia da Mulher da Sociedade Brasileira de Cardiologia - 2020. Arquivos Brasileiros de Cardiologia, 115(5), 975-986.

Méndez, D.N, \& Núñez, D.C. (2011). Enfermedad tromboembólica venosa en el embarazo y puerperio. Enfoque de riesgo y diagnóstico. MEDISAN, 15(10), $1423-1441$.

Méndez, D. N, \& Núñez, D. C. (2011). Experiencia de 11 años en la enfermedad tromboembólica venosa en el período grávido puerperal. Revista Cubana de Obstetricia y Ginecología, 37(3), 300-311.

Montenegro, C. A. B., \& Filho, J. R. (2018). Obstetrícia Fundamental (14th ed.). Guanabara Koogan.

Oliveira, A. L. M. L., \& Maques, M. A. (2016). Profilaxia de tromboembolismo venoso na gestação. Jornal Vascular Brasileiro, 15(4), $293-301$.

Oliveira, K., Oliveira, J., Wernet, M., Paschoini, M., \& Ruiz, M. (2021). Período pós-parto e infecção pelo novo coronavírus: revisão de escopo. Revista Enfermagem UERJ, 29(1), e56037.

Pontes, D. M., Pimentel, L. G. B., \& Carvalho, F. H. C. (2013). Eventos tromboembólicos na gestação e puerpério: revisão sistemática e recomendação atual. Revista Feminina, 41(1).

Ramírez-Calderón, F., Vásquez-Alva, R., Lazo-Gordillo, D.E., \& Zamora-Chávez, S.C. (2018). Trombosis venosa profunda de miembro inferior en gestante del primer trimestre: Reporte de caso. Revista Peruana de Ginecología y Obstetricia, 64(3), 473-478.

Roever, L. (2017). Compreendendo os estudos de revisão sistemática. Rev. Soc. Bras. Clin. Med, 15(2), 127-130.

Rojas-Sanchez, A. G., Rosa, G. N., Mijangos-Mendez, J. C., \& Campos-Cerda, R. (2014). Tromboembolia pulmonar en el embarazo y puerperio. Neumol. Cir. Torax, 73(1), 42-48.

Servante, J., Swallow, G., Thornton, J. G., Myers, B., Munireddy, S., Malinowski, A. K., Othman, M., Li, W., O'Donoghue, K., \& Walker, K. F. (2021). Haemostatic and thrombo-embolic complications in pregnant women with COVID-19: a systematic review and critical analysis. BMC pregnancy and childbirth, 21(1), 108 .

Yang, H., Wang, C., \& Poon, L. C. (2020). Novel coronavirus infection and pregnancy. Ultrasound in obstetrics \& gynecology : the official journal of the International Society of Ultrasound in Obstetrics and Gynecology, 55(4), 435-437. 